\title{
The Role of Long Noncoding RNAs in Patients With Luminal A Invasive Breast Ductal Carcinoma
}

\author{
Nahal Eshghifar \\ Islamic Azad University \\ Fatemeh Rouhollah \\ Islamic Azad University \\ Nooshin Barikrow \\ Islamic Azad University \\ Farkhondeh Pouresmaeili ( $\sim$ Pouresfar@gmail.com ) \\ Shahid Beheshti University of Medical Sciences \\ Mohammad Taheri \\ Shahid Beheshti University of Medical Sciences
}

\section{Research Article}

Keywords: LncRNA, Breast cancer, prognosis, Quantitative Real-Time PCR

Posted Date: June 24th, 2021

DOI: https://doi.org/10.21203/rs.3.rs-625557/v1

License: (c) (i) This work is licensed under a Creative Commons Attribution 4.0 International License.

Read Full License 


\section{Abstract}

Breast cancer is the most common form of cancer in women around the world. The molecular mechanisms of this heterogeneous disease have been extensively investigated; but still; need many sensitive and specific markers for prognostic and early diagnostic approaches. Non-protein coding RNAs known as IncRNAs are reported in tumorogenesis involvement. hence could be used as therapeutic targets.

In the present study, we examined the expression levels of CCAT1, PDCD4, PDCD4-AS1, and MEG3 LncRNAs in tumor and adjacent breast tissues in 88 Iranian patients by quantitative real-time PCR. CCAT1 was notably overexpressed and PDCD4-AS1 was decreased in tumor samples, PDCD4 and PDCD4-AS1 showed a positive association with each other, higher levels of PDCD4-AS1 were associated with better survival, tumor samples showed lower levels of PDCD4 compared to normal tissue.

Our findings show that IncRNAs play critical roles in controlling post-transcriptional gene expression of key tumor suppressor or oncogenic genes, leading to TNBC progression.

As a result, this research looked into the prognostic role of IncRNAs in breast cancer patients.

\section{Introduction}

Breast cancer (BC), the main cause of world cancer-related deaths in women, may be a popular and highly mortal malignancy $(1,2)$.

As identifying molecular factors for $\mathrm{BC}$ treatment may help advance survival rates, further researches have attempted to find biological targets which might be utilized for early prognostic and diagnosis approaches $(3,4)$. However, few genes have been detected as prognostic or therapeutic biomarkers of BC. Among all breast cancer subtypes, Luminal $A$ has the finest prognosis and indicates low grade, low cell proliferation, and high sensitivity to endocrine therapy(5). To be cited by end note

Long non-coding RNAs (IncRNAs) are commonly more than 200 nucleotides in length and could not be encoded into proteins. However, IncRNAs have been shown to play critical regulatory roles in apoptosis, cell proliferation, differentiation, and migration in previous studies $(6,7)$. Multiple types of research demonstrate the correlation between IncRNA expression and survival, metastasis formation, and clinical recurrence $(8,9)$. MEG3 is one of the IncRNAs that are consistently down-regulated in malignancies of different types of tissue origins, such as lung cancer(10), cervical cancer(11), pituitary tumor(12) , and uterus cancer(13). However, the prognostic importance of MEG3 in BC is still unknown.

Programmed cell death, or Apoptosis, may be a crucial biological mechanism, which occurs during the diverse process from growth to tissue turnover(14). One of the cell's protective mechanisms against tumor growth is the elimination of defective cells with abnormal gene expression and apoptosis(15). Any 
deficiency in apoptosis can induce diseases such as cancer, and resistance to apoptosis is one of the key characteristics of cancerous cells (16).

As a result, the expression of apoptosis-related genes is closely regulated. One of the apoptosisassociated genes is programmed cell death 4 (PDCD4), which is up-regulated after apoptosis initiation. PDCD4-AS1 could be a two-exon transcript related to a genetic material on chromosome 10q24(17). PDCD4-AS1 is a long non-coding RNA, which is encoded by the complementary strand of Programmed Cell Death 4 (PDCD4). The tumor suppressor gene PDCD4 is well-known to negatively regulate tumor invasion, neoplastic transformation ,and cell proliferation (18) While PDCD4-AS1 lacks protein-coding potential, it is encoded in the opposite direction as the protein-coding gene known as PDCD4(19). Unlike PDCD4-AS1 has not been studied yet. PDCD4 is a very well-known tumor suppressor gene(20). CCAT1 has been appeared to be over expressed in various cancers, including, hepatocellular carcinoma, gastric cancer, gallbladder cancer, and colorectal cancer (21-23). However, the therapeutic importance of CCAT1 in $\mathrm{BC}$ has not been investigated yet. The LncRNAs supporting the functions of promoting tumor invasion and metastasis have shown in Table 1.

More understanding of the molecular pathway of Luminal A breast cancer and its related genetic disorders could help us to recognize prognostic and diagnostic biomarkers and effective therapy. In this research, the molecular mechanism underlying breast cancer, focusing on the luminal A subtype with the expression levels of 4 IncRNAs in tumor cells and adjacent normal tissues were examined. 
Table 1

LncRNAs supporting the functions of promoting tumor invasion and metastasis

\begin{tabular}{|c|c|c|c|c|}
\hline LncRNAs & $\begin{array}{l}\text { Source (Tissues } \\
\text { and/or cells) }\end{array}$ & $\begin{array}{l}\text { Expression } \\
\text { in patients }\end{array}$ & Experimental data & Reference \\
\hline CCAT1 & $\begin{array}{l}\text { HCC, GC, Breast } \\
\text { cancer }\end{array}$ & Increased & $\begin{array}{l}\text { Promote cell proliferation, } \\
\text { increase invasion and } \\
\text { metastasis }\end{array}$ & $(22,23)$ \\
\hline MEG3 & $\begin{array}{l}\text { AML, Bladder, CML, } \\
\text { Colon, } \\
\text { Gastric, Glioma, } \\
\text { Hepatocellular, } \\
\text { Kidney, Lung, } \\
\text { Meningioma, } \\
\text { Neuroblastoma, } \\
\text { Prostate }\end{array}$ & Decreased & $\begin{array}{l}\text { Regulating the TGF-beta Pathway } \\
\text { genes through formation of RNA- } \\
\text { DNA triplex structures }\end{array}$ & (24) \\
\hline PDCD4 & $\begin{array}{l}\text { Colorectal, Lung, } \\
\text { Ovarian, Hepatoma } \\
\text { cells }\end{array}$ & Decreased & $\begin{array}{l}\text { identified in a screen aimed to } \\
\text { determine } \\
\text { apoptosis-induced targets }\end{array}$ & $(25-27)$ \\
\hline $\begin{array}{l}\text { PDCD4- } \\
\text { AS1 }\end{array}$ & Ovarian, Colorectal & Decreased & $\begin{array}{l}\text { it regulates the expression of its } \\
\text { sense protein-coding partner, } P D C D 4\end{array}$ & $(28,29)$ \\
\hline
\end{tabular}

\section{Materials And Methods}

\section{Patients and tissue specimens}

In this study 88 pairs of fresh breast cancer specimens, type luminal A, and adjacent noncancerous tissues were taken from the same patients in Hamadan Hospital, collected in liquid nitrogen and stored in $-70^{\circ} \mathrm{C}$ until use for RNA extraction. The adjacent tissue samples were characterized and confirmed to be normal by the referred hospital pathologists. None of the patients had received preoperative radiotherapy or chemotherapy. Patients' demographic information including their clinico-pathological was considered. They also provided a written informed consent form for tissue specimen analysis.

We confirm that all experimental protocols were approved by the Ethics committee of Shahid Beheshti University of Medical Sciences and all methods were carried out in accordance with relevant guidelines and regulations

\section{RNA extraction and RT-qPCR analysis}

Total cellular RNA was extracted from the under study tissues utilizing Trizol reagent (Invitrogen, Waltham, MA, USA) and quantified with Nanodrop 2000 using manufacturer's protocol for concentration 
and purity. Agarose gel electrophoresis was used to identify extracted nucleic acids integrity. Then, one microgram RNA was used to be reversely encoded into cDNA by Random hexamers and Multiscribe reverse transcriptase (Applied Biosystems) and quantified by following spectrophotometry. LncRNA expression levels were evaluated by Quantitative RT-PCR using the StepOne Plus system and SYBR Green PCR Master Mix according to the following cycling parameters, $95^{\circ} \mathrm{C}$ for $10 \mathrm{~min}$, followed by 40 cycles of $95^{\circ} \mathrm{C}$ for $15 \mathrm{~s}$ and $60^{\circ} \mathrm{C}$ for $45 \mathrm{~s}$. A negative control was used in each run. The housekeeping gene HPRT1 was chosen for normalization. The PCR primer sequences for the selected LncRNAs were listed as follows:

CCAT1:

Forward primer $\quad$ 5'-TCACTGACAACATCGACTTTGAAG-3'

Reverse primer 5'-GGAGAAAACGCTTAGCCATACAG-3'

PDCD4:

Forward primer 5'-TCTGGGAAAGGAAGGGGACTAC-3'

Reverse primer $\quad$ 5'-TTCATAAACACAGTTCTCCTGGTCAT-3'

PDCD4-AS1:

Forward primer 5'-GGTCAGTGGCCTAGTGAGC-3'

Reverse primer $\quad$ 5'CAGTCTAATGGGCAGAAGGGC3'

\section{MEG3:}

Forward primer $\quad$ 5'- GCTGGGTCGGCTGAAGAAC-3'

Reverse primer 5'-CGTGGCTGTGGAGGGATTT-3'

\section{HPRT1}

Forward primer $\quad$ 5'- CACTATATTGCCCAGGTTGGT- 3 '

Reverse primer $\quad 5^{\prime}$ - GCGGAAGCGTGTAAAATC -3' 
To evaluate the IncRNA relative expression in adjacent normal tissues in comparison with BC tissues, we used melting temperatures, respectively. Each sample was analyzed in duplicate.

\section{Statistical Analysis}

Statistical analysis was performed utilizing the SPSS version 24.0 (SPSS, Chicago, IL, USA).

We used Bayesian estimation supersedes the t-test (BEST) to test the significance of difference in means between two paired groups. A t student prior family was assumed for parameters with 4000 iterations and 2000 burn-outs. The $P$ values estimated from Frequentist methods including median test.

The Spearman correlation was used to assess the association among relative gene expressions. We used BEST, Rjags, and ggplot2 packages to implement analysis in the software R 4.03. A p-value $<0.05$ was considered statistically significant.

\section{Results}

The expression level of LncRNAs considered in this study was measured to evaluate their potential in diagnostic tests. The ROC curves of each were plotted. The area below each curve was compared and evaluated using the sensitivity and specificity of each molecule. Values above 0.9 were considered excellent (Mandrekar, 2010).

Simultaneous expression of none of the studied genes was significantly associated with metastasis. The expression of all genes was statistically significant between the two groups of healthy and tumors. Based on t-Test analysis, all studied genes had completely different expression in healthy and involved tissues; expression was higher in healthy tissues (Table2). CCAT1 showed higher sensitivity than the other examined genes (ROC CURVES, Fig. 1).

PDCD4-AS1 is decreased during breast cancer development, and its interactions are positively associated with PDCD4 in BC tissue. Also, PDCD4 and PDCD4-AS1 indicated a remarkable positive association with each other in the breast cancer patients. Eventually, the higher levels of PDCD4-AS1 were associated with better survival value in a cohort of BC patients. Similar to PDCD4-AS1, patient samples showed lower levels of PDCD4 compared to normal tissue (Fig. 2,3).

In comparison with adjacent normal tissues, No expression of MEG3 was observed in understudied samples, so it was removed from the study. The relative gene expression comparison between tumor and healthy samples; Results of Bayesian estimation supersedes the t-test have been shown in Table 2 . 
Table 2

The comparison relative gene expression between tumor and healthy samples; Results of Bayesian estimation supersedes the t-test.

\begin{tabular}{|llllll|}
\hline Gene & Posterior mean diff. & SD & Effect size & P-value & $95 \%$ HDI \\
\hline CCAT1 & -4.944 & 3.05 & -1.686 & $<0.0001$ & {$[-6.08,-3.81]$} \\
\hline PDCD4 & -1.92 & 2.77 & -0.719 & $<0.0001$ & {$[-2.95,-0.89]$} \\
\hline PDCD4-AS1 & -0.97 & 2.15 & -0.461 & 0.007 & {$[-1.83,-0.14]$} \\
\hline *Abbreviations; SD: Standard deviation, HDI: highest density interval & \\
\hline
\end{tabular}

\section{Discussion}

An increasing number of novel therapeutic methods have been improved for Breast cancer, such as molecular immunotherapy, gene therapy, and targeted treatment. However, satisfactory therapeutic methods have not been obtained yet. Breast cancers have remained a main public health issue. Recently, multiple kinds of research have been conducted to investigate the function of dysregulated IncRNA expression in different kinds of human cancer types such as BC $(30,31)$ we have attempted to evaluate the molecular role of differentially expressed IncRNAs, to better understand the process leading breast cancer progression

Our results revealed that MEG3 had no expression either in healthy or in tumor tissues. Recent researches have shown that, this IncRNA plays an important role in the progression of various disorders, including cancer. In the research of Luo et al(32) MEG3 played an important function in controlling migration, apoptosis, and cell proliferation by targeting Bcl-2 in prostate cancer. Sun et al(33) implied that MEG3 might be a poor prognostic indicator in BC and knockdown of MEG3 by siRNA may induce BC cells proliferation apoptosis. Braconi et al(34) showed that ectopic expression level of MEG3 induced apoptosis in hepatocellular cancer cells. Sun et. al (13) revealed that decreasing MEG3 inhibited proliferation, invasion , and migration by relying on p53's transcriptional function. All of the data presented above implied that MEG3 might be a novel molecular marker associated with BC progression and under the regulation of a population depended genomic network of polymorphisms where it plays an independent prognostic role as a biomarker as well as a new potential indicator for $\mathrm{BC}$ patients in particular or ethnic populations. Further researches are needed to decide whether MEG3 expression might be considered as a potential $\mathrm{BC}$ biomarker and if it affects the survival rate of $\mathrm{BC}$ patients in Iranian ethnicity.

Recent studies have demonstrated that CCAT1 was overexpressed in various types of cancers and was involved in a variety of cellular mechanisms associated with carcinogenesis. However, the prognostic and clinical importance values of CCAT1 in breast cancer haven't been investigated.

According to He et al studies CCAT1 has shown to be remarkably up-regulated in colorectal cancer in comparison with adjacent normal tissues and its over expressed expression was associated with 
patients' clinical stage, metastasis of lymph nodes, and survival rate after surgery. Furthermore, c-Myc might directly induce CCAT1 transcription by binding to its promoter, and enhancement of CCAT1 expression in colorectal cancer induced invasion and cell proliferation (35).

In the study of Yang et al CCAT1 was significantly over-expressed in gastric cancer; c-Myc induction of CCAT1 plays a substantial role in gastric cancer and suggest that CCAT1 maybe used to treat gastric cancer(22).

In the present study, we distinguished that CCAT1 expression was notably higher in adjacent normal tissues than in BC patients [p-value $<0.0001 ; 87.8 \%$ sensitivity, $90 \%$ specificity, $95 \% \mathrm{Cl}$ for cut-off point $(>-0.73,>1.35)]$. The correlations of the CCAT1 with diverse clinical characteristics of BC were evaluated. We found that over expression of CCAT1 was not associated with lymph node metastasis, implying that CCAT1 could be involved in the aggressive disorder and carcinogenesis in BC. More analysis indicated that over expression of CCAT1 is an independent prognosis biomarker for overall survival and progression-free survival.

More researches in to the molecular pathway by which CCAT1 contributes to the onset and BC progression are required.

Moreover, this study evaluated the expression and clinical importance of both PDCD4-AS1 and PDCD4. They were chosen for mechanistic researches for the following reasons. First, PDCD4 is previously introduced as a tumor suppressor gene, that is down regulated in different types of cancer $(36,37)$. Second, PDCD4 and PDCD4-AS1 are expressed in both luminal A patient specimens and BC cell lines. Eventually, clinical survival evidence from BC patients demonstrates that similar to the PDCD4 gene, down-regulation of PDCD4-AS1 decreased patient survival rate, indicating a tumor suppressor function of PDCD4-AS1. The results of our research show that PDCD4-AS1 such as PDCD4 has significantly decreased expression in tumor tissues ( $p$-value 0.007 ) and that the levels of PDCD4 and PDCD4-AS1 are co-regulated in BC patients. Better survival of patients with higher levels of PDCD4-AS1 and Low levels of PDCD4-AS1 in BC samples suggests that PDCD4-AS1, similar to its sense partner PDCD4, may act as a tumor suppressor gene. With these observations, we encounter a greater complexity of PDCD4-AS1 function in regulation and increase of PDCD4 cells' level during the progression of breast cancer, which requires further investigation in a larger number of samples.

Therefore, discovering essential IncRNA expression signatures in BC progression will be helpful in the identification of novel prognostic and diagnostic biomarkers for BC therapy. Future researches are required to identify the mechanistic function of other BC dysregulated IncRNAs in breast cancer biology.

\section{Declarations}

\section{Data availability}

Data are available from the corresponding author on reasonable request 


\section{Author Contributions}

F.P.; Designed analyzed data, co-wrote the paper and Critical revision of the manuscript for important intellectual content; M.T; Study concept and design, Acquisition of data; collecting samples, N.E; Acquisition of data, performed the searches ,co-wrote the paper, Performed proof editing,; F.R: Analysis and interpretation of data, Critical revision of the manuscript for important intellectual content. N.B: Critical revision of the manuscript for important intellectual content, Performed proof editing. All the authors provided their final approval for the completed manuscript.

\section{Ethics declarations}

\section{Conflict of interest}

The authors declare no conflicts of interest.

\section{References}

1. Murray PJ, Wivell G, Denton E. Breast cancer screening and diagnosis in the 21 st century within the UK. Post reproductive health. 2015;21(3):105-11.

2. Siegel RL, Miller KD, Jemal A. Cancer statistics, 2016. CA: a cancer journal for clinicians. 2016;66(1):730.

3. Khanfir A, Lahiani F, Bouzguenda R, Ayedi I, Daoud J, Frikha M. Prognostic factors and survival in metastatic breast cancer: a single institution experience. Reports of Practical Oncology \& Radiotherapy. 2013;18(3):127-32.

4. Wang C, Yuan P, Xu B, Yuan L, Yang H, Liu X. RLIP76 expression as a prognostic marker of breast cancer. Eur Rev Med Pharmacol Sci. 2015;19(11):2105-11.

5. Gao JJ, Swain SM. Luminal a breast cancer and molecular assays: a review. The oncologist. 2018;23(5):556.

6. Hu W, Alvarez-Dominguez JR, Lodish HF. Regulation of mammalian cell differentiation by long noncoding RNAs. EMBO reports. 2012;13(11):971-83.

7. Gutschner T, Diederichs S. The hallmarks of cancer: a long non-coding RNA point of view. RNA biology. 2012;9(6):703-19.

8. Tuo Y, Li X, Luo J. Long noncoding RNA UCA1 modulates breast cancer cell growth and apoptosis through decreasing tumor suppressive miR-143. Eur Rev Med Pharmacol Sci. 2015;19(18):3403-11.

9. Liao X, Wang J, Li L, Zhou D, Ren K, Jin Y, et al. Long intergenic non-coding RNA APOC1P1-3 inhibits apoptosis by decreasing a-tubulin acetylation in breast cancer. Cell death \& disease. 2016;7(5):e2236-e. 
10. Lu K-h, Li W, Liu X-h, Sun M, Zhang M-I, Wu W-q, et al. Long non-coding RNA MEG3 inhibits NSCLC cells proliferation and induces apoptosis by affecting p53 expression. BMC cancer. 2013;13(1):1-11.

11. Zhang J, Yao T, Wang Y, Yu J, Liu Y, Lin Z. Long noncoding RNA MEG3 is downregulated in cervical cancer and affects cell proliferation and apoptosis by regulating miR-21. Cancer biology \& therapy. 2016;17(1):104-13.

12. Chunharojrith $P$, Nakayama $Y$, Jiang $X$, Kery RE, Ma J, Cristine S, et al. Tumor suppression by MEG3 IncRNA in a human pituitary tumor derived cell line. Molecular and cellular endocrinology. 2015;416:2735.

13. Sun L, Li Y, Yang B. Downregulated long non-coding RNA MEG3 in breast cancer regulates proliferation, migration and invasion by depending on p53's transcriptional activity. Biochemical and biophysical research communications. 2016;478(1):323-9.

14. Ke B, Tian M, Li J, Liu B, He G. Targeting programmed cell death using small-molecule compounds to improve potential cancer therapy. Medicinal research reviews. 2016;36(6):983-1035.

15. Ouyang L, Shi Z, Zhao S, Wang FT, Zhou TT, Liu B, et al. Programmed cell death pathways in cancer: a review of apoptosis, autophagy and programmed necrosis. Cell proliferation. 2012;45(6):487-98.

16. Hanahan D, Weinberg RA. Hallmarks of cancer: the next generation. cell. 2011;144(5):646-74.

17. Jadaliha M, Gholamalamdari O, Tang W, Zhang Y, Petracovici A, Hao Q, et al. A natural antisense IncRNA controls breast cancer progression by promoting tumor suppressor gene mRNA stability. PLoS genetics. 2018;14(11):e1007802.

18. Lankat-Buttgereit B, Göke R. The tumour suppressor Pdcd4: recent advances in the elucidation of function and regulation. Biology of the Cell. 2009;101(6):309-17.

19. Wang D, Wang Z, Zhang L, Sun S. LncRNA PDCD4-AS1 alleviates triple negative breast cancer by increasing expression of IQGAP2 via miR-10b-5p. Translational oncology. 2021;14(1):100958.

20. Sadeq S, Al-Hashimi S, Cusack CM, Werner A. Endogenous Double-Stranded RNA. Non-coding RNA. 2021;7(1):15.

21. Nissan A, Stojadinovic A, Mitrani-Rosenbaum S, Halle D, Grinbaum R, Roistacher M, et al. Colon cancer associated transcript-1: A novel RNA expressed in malignant and pre-malignant human tissues. International journal of cancer. 2012;130(7):1598-606.

22. Yang F, Xue X, Bi J, Zheng L, Zhi K, Gu Y, et al. Long noncoding RNA CCAT1, which could be activated by c-Myc, promotes the progression of gastric carcinoma. Journal of cancer research and clinical oncology. 2013;139(3):437-45. 
23. Deng L, Yang S-B, Xu F-F, Zhang J-H. Long noncoding RNA CCAT1 promotes hepatocellular carcinoma progression by functioning as let-7 sponge. Journal of experimental \& clinical cancer research. 2015;34(1):1-10.

24. Mondal T, Subhash S, Vaid R, Enroth S, Uday S, Reinius B, et al. MEG3 long noncoding RNA regulates the TGF- $\beta$ pathway genes through formation of RNA-DNA triplex structures. Nature communications. 2015;6(1):1-17.

25. Allgayer $\mathrm{H}$. Pdcd4, a colon cancer prognostic that is regulated by a microRNA. Critical reviews in oncology/hematology. 2010;73(3):185-91.

26. Hu Y, Wei X, Lv Y, Xie X, Yang L, He J, et al. EIF3H interacts with PDCD4 enhancing lung adenocarcinoma cell metastasis. American journal of cancer research. 2020;10(1):179.

27. Li H, Xu H, Shen H. microRNA-106a modulates cisplatin sensitivity by targeting PDCD4 in human ovarian cancer cells. Oncology letters. 2014;7(1):183-8.

28. Wang $J$, Tian $Y$, Zheng $H$, Ding $Y$, Wang $X$. An integrated analysis reveals the oncogenic function of IncRNA LINC00511 in human ovarian cancer. Cancer medicine. 2019;8(6):3026-35.

29. Akbari F, Peymani M, Salehzadeh A, Ghaedi K. Integrative in silico and in vitro transcriptomics analysis revealed new IncRNAs related to intrinsic apoptotic genes in colorectal cancer. Cancer cell international. 2020;20(1):1-16.

30. Spizzo R, Almeida Mle, Colombatti A, Calin GA. Long non-coding RNAs and cancer: a new frontier of translational research? Oncogene. 2012;31(43):4577-87.

31. Yang Z, Zhou L, Wu L-M, Lai M-C, Xie H-Y, Zhang F, et al. Overexpression of long non-coding RNA HOTAIR predicts tumor recurrence in hepatocellular carcinoma patients following liver transplantation. Annals of surgical oncology. 2011;18(5):1243-50.

32. Luo G, Wang M, Wu X, Tao D, Xiao X, Wang L, et al. Long non-coding RNA MEG3 inhibits cell proliferation and induces apoptosis in prostate cancer. Cellular physiology and biochemistry. 2015;37(6):2209-20.

33. Sun M, Xia R, Jin F, Xu T, Liu Z, De W, et al. Downregulated long noncoding RNA MEG3 is associated with poor prognosis and promotes cell proliferation in gastric cancer. Tumor Biology. 2014;35(2):1065-73.

34. Braconi C, Kogure T, Valeri N, Huang N, Nuovo G, Costinean S, et al. microRNA-29 can regulate expression of the long non-coding RNA gene MEG3 in hepatocellular cancer. Oncogene. 2011;30(47):4750-6.

35. He X, Tan X, Wang X, Jin H, Liu L, Ma L, et al. C-Myc-activated long noncoding RNA CCAT1 promotes colon cancer cell proliferation and invasion. Tumor Biology. 2014;35(12):12181-8. 
36. Ma G, Zhang H, Dong M, Zheng X, Ozaki I, Matsuhashi S, et al. Downregulation of programmed cell death 4 (PDCD4) in tumorigenesis and progression of human digestive tract cancers. Tumor Biology. 2013;34(6):3879-85.

37. Ding X, Cheng X, Gong M, Chen X, Yin F, Lai K. Hypermethylation and expression silencing of PDCD4 gene in hepatocellular carcinoma: a consort study. Medicine. 2016;95(6).

\section{Figures}

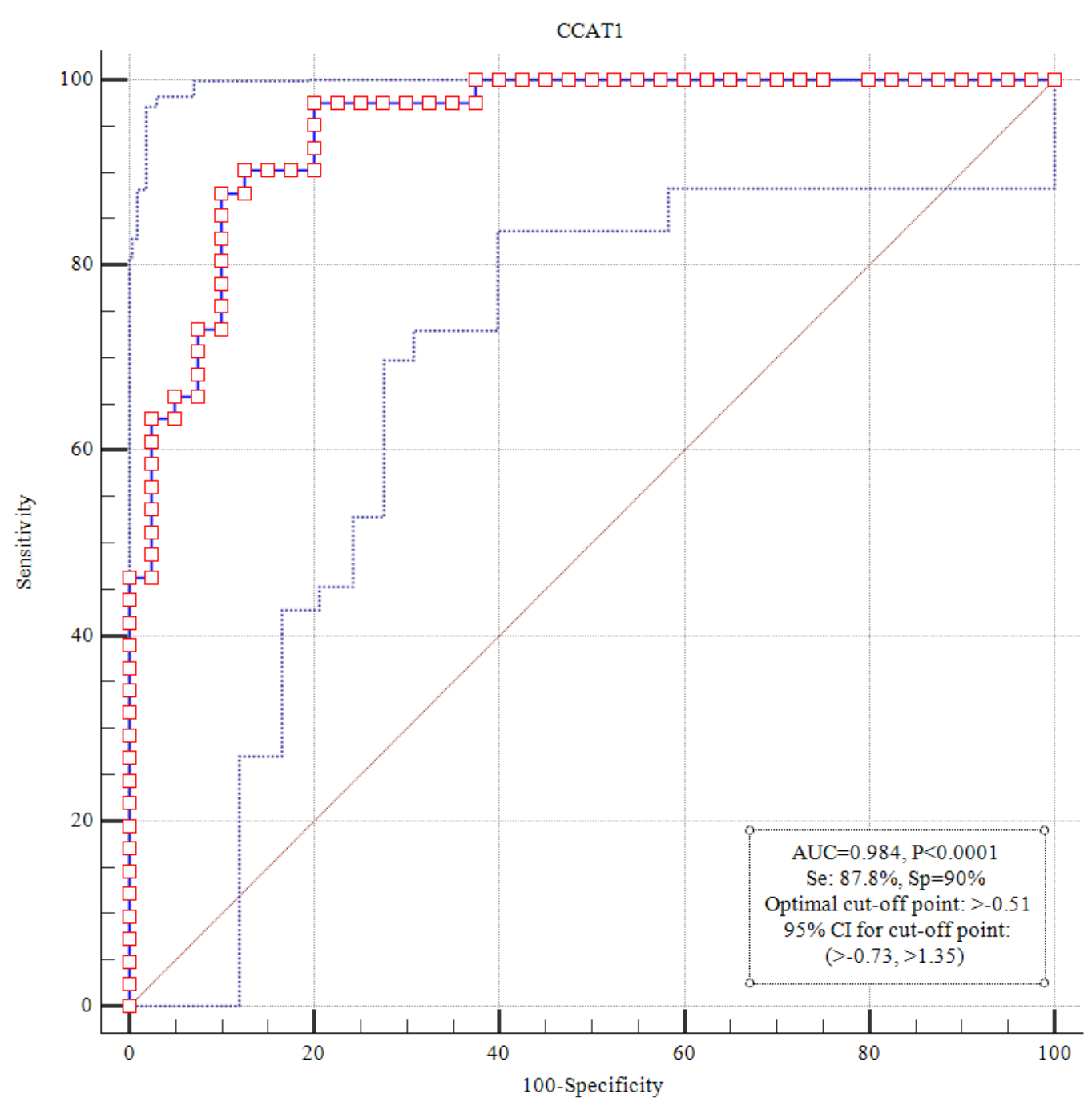

\section{Figure 1}

Receiver operating characteristics curves showing the accuracy of CCAT1 in distinguishing between Breast cancers (Luminal A) Patients and healthy ones 


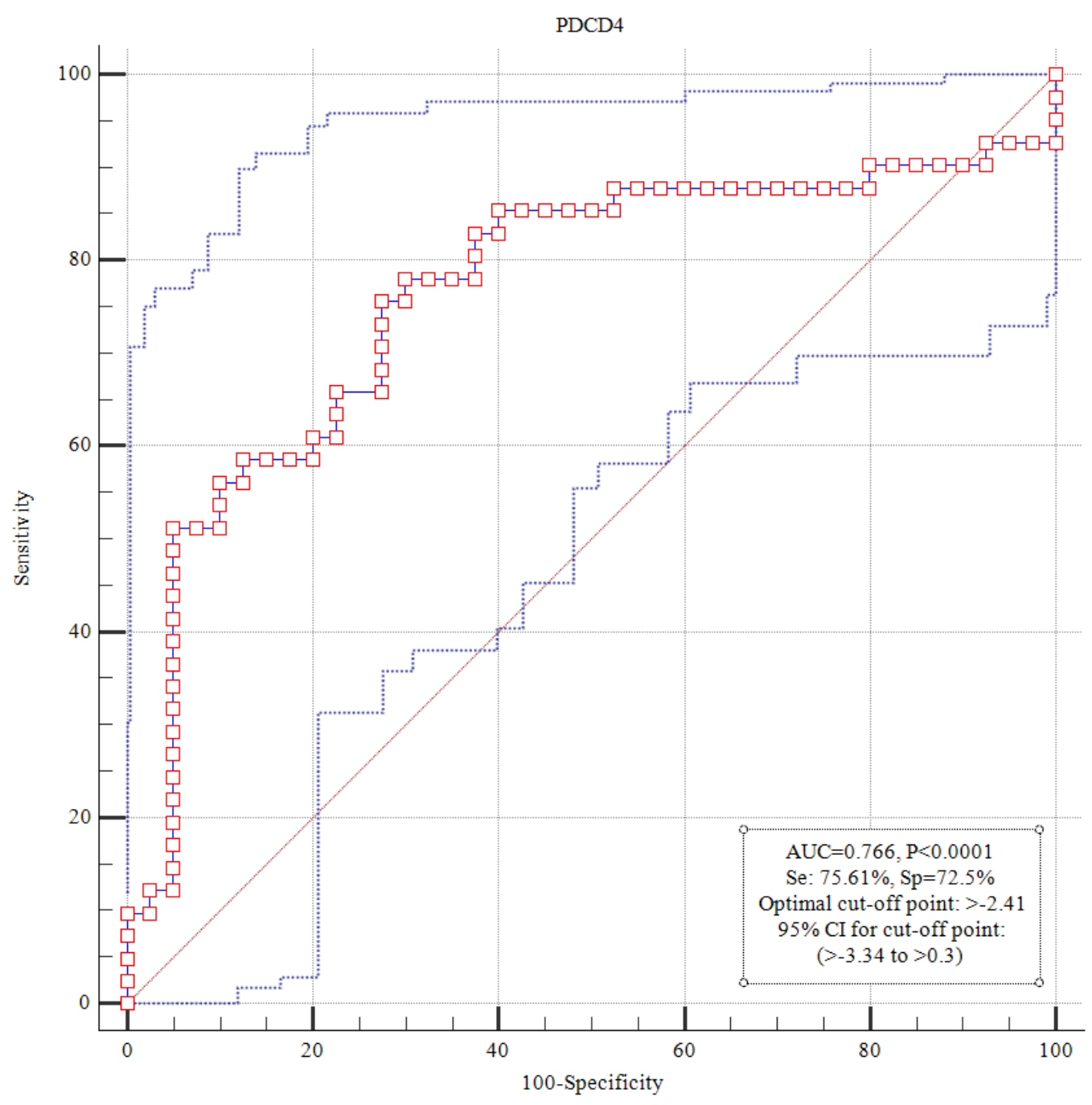

Figure 2

Receiver operating characteristics curves showing the accuracy of PDCD4 in distinguishing between Breast cancers (Luminal A) Patients and healthy ones 


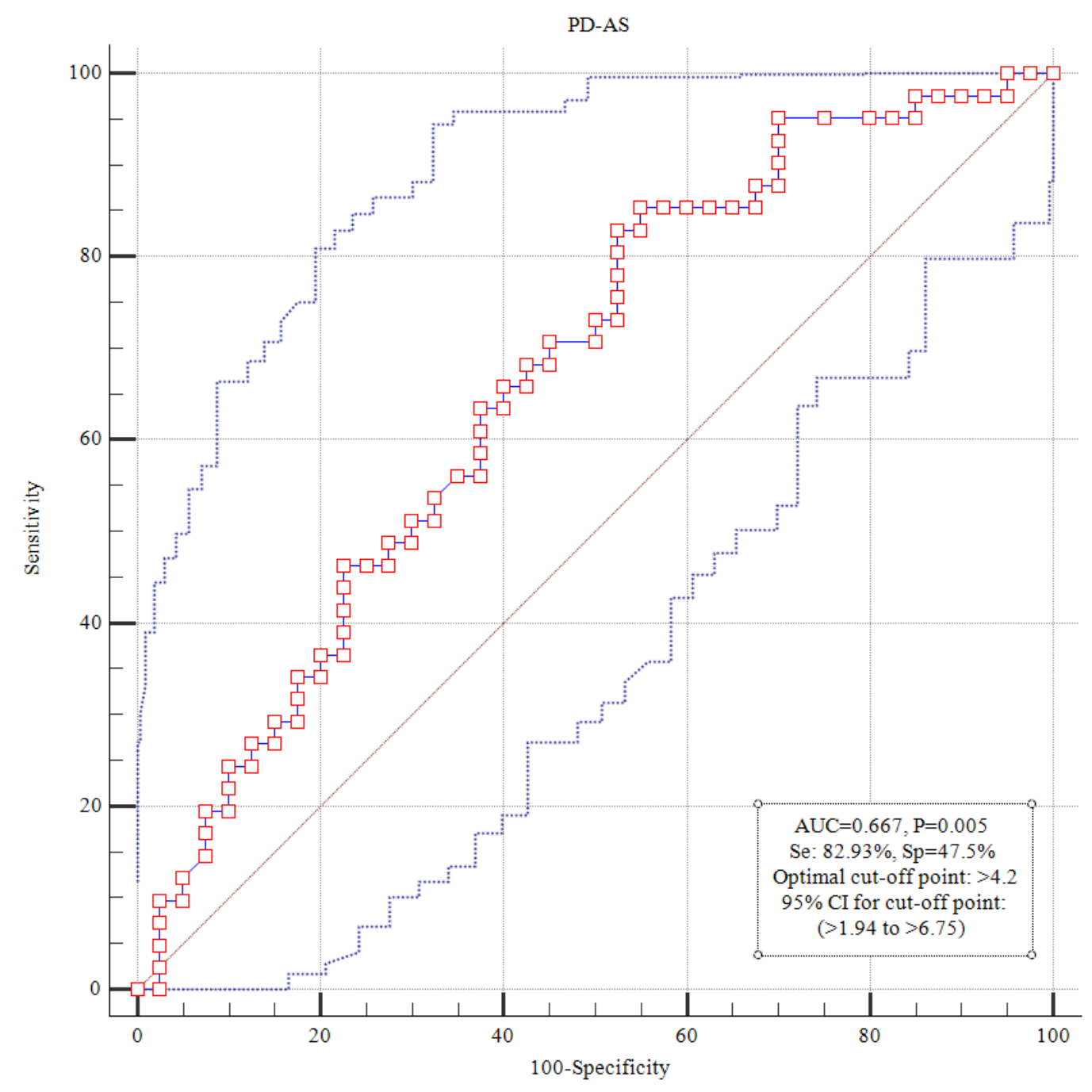

Figure 3

Receiver operating characteristics curves showing the accuracy of PDCD4-AS1 in distinguishing between Breast cancers (Luminal A) Patients and healthy ones 